\title{
Some identities on the higher-order-twisted $q$-Euler numbers and polynomials with weight $\alpha$
}

\author{
Hui Young Lee*, Nam Soon Jung, Jung Yoog Kang and Cheon Seoung Ryoo
}

\author{
* Correspondence: normaliz@hnu.kr \\ Department of Mathematics, \\ Hannam University, Daejeon 306- \\ 791, Korea
}

\begin{abstract}
In this article, we introduce some properties of higher-order-twisted $q$-Euler numbers and polynomials with weight $\alpha$, and we observe some properties of higher-ordertwisted $q$-Euler numbers and polynomials with weight $\alpha$ for several cases. In particular, by using the the fermionic $p$-adic $q$-integral on $\mathbb{Z}_{p}$, we give a new concept of twisted $q$-Euler numbers and polynomials with weight $\alpha$.

2000 Mathematics Subject Classification: 11B68; 11S40; 11580.
\end{abstract}

Keywords: Euler numbers and polynomials, q-Euler numbers and polynomials, higher-order-twisted $q$-Euler numbers and polynomials with weight $a$

\section{Introduction}

Let $p$ be a fixed odd prime. Throughout this article $\mathbb{Z}_{p}, \mathbb{Q}_{p}, \mathbb{C}$, and $\mathbb{C}_{p}$, will, respectively, denote the ring of $p$-adic rational integers, the field of $p$-adic rational numbers, the complex number field, and the completion of algebraic closure of $\mathbb{Q}_{p}$. Let $\mathbb{N}$ be the set of natural numbers and $\mathbb{Z}_{+}=\mathbb{N} \cup\{0\}$. Let $v_{p}$ be the normalized exponential valuation of $\mathbb{C}_{p}$ with $|p|_{p}=p^{-v_{p}(p)}=p^{-1}$ (see [1-14]). When one speaks of $q$-extension, $q$ can be regarded as an indeterminate, a complex number $q \in \mathbb{C}$, or $p$-adic number $q \in \mathbb{C}_{p}$; it is always clear from context. If $q \in \mathbb{C}$, we assume $|q|<1$. If $q \in \mathbb{C}_{p}$, then we assume $\mid$ $1-\left.q\right|_{p}<1$ (see [1-14]).

In this article, we use the notation of $q$-number as follows (see [1-14]):

$$
[x]_{q}=\frac{1-q^{x}}{1-q} .
$$

Note that $\lim _{q \rightarrow 1}[x]_{q}=x$ for any $x$ with $|x|_{p} \leq 1$ in the $p$-adic case.

Let $C\left(\mathbb{Z}_{p}\right)$ be the space of continuous functions on $\mathbb{Z}_{p}$. For $f \in C\left(\mathbb{Z}_{p}\right)$, Kim defined the fermionic $p$-adic $q$-integral on $\mathbb{Z}_{p}$ as follows (see $[6,7]$ ):

$$
\begin{aligned}
I_{-q}(f)=\int_{\mathbb{Z}_{p}} f(x) d \mu_{-q}(x) & =\lim _{N \rightarrow \infty} \frac{1}{\left[p^{N}\right]_{-q}} \sum_{x=0}^{p^{N}-1} f(x)(-q)^{x} \\
& =\lim _{N \rightarrow \infty} \frac{[2]_{q}}{1+q^{p^{N}}} \sum_{x=0}^{p^{N}-1} f(x)(-q)^{x} .
\end{aligned}
$$

\section{Springer}

(c) 2012 Lee et al; licensee Springer. This is an Open Access article distributed under the terms of the Creative Commons Attribution License (http://creativecommons.org/licenses/by/2.0), which permits unrestricted use, distribution, and reproduction in any medium, provided the original work is properly cited. 
From (1), we note that

$$
q I_{-q}\left(f_{1}\right)+I_{-q}(f)=[2]_{q} f(0),
$$

where $f_{1}(x)=f(x+1)$.

It is well known that the ordinary Euler polynomials are defined by

$$
\frac{2}{e^{t}+1} e^{x t}=e^{E(x) t}=\sum_{n=0}^{\infty} E_{n}(x) \frac{t^{n}}{n !}
$$

with the usual convention of replacing $E^{n}(x)$ by $E_{n}(x)$.

In the special case, $x=0, E_{n}(0)=E_{n}$ are called the $n$th Euler numbers (see [1-14]).

By (2), we get the following recurrence relation as follows:

$$
E_{0}=1, \text { and }(E+1)^{n}+E=\left\{\begin{array}{l}
2, \text { if } n=0 \\
0, \text { if } n>0
\end{array}\right.
$$

Recently, $(h, q)$-Euler numbers are defined by

$$
E_{0, q}^{(h)}=\frac{2}{1+q^{h}}, \text { and } q^{h}\left(q E_{q}^{(h)}+1\right)^{n}+E_{q}^{(h)}=\left\{\begin{array}{l}
2, \text { if } n=0, \\
0, \text { if } n>0
\end{array}\right.
$$

with the usual convention about replacing $\left(E_{q}^{(h)}\right)^{n}$ by $E_{n, q}^{(h)}($ see [1-16]).

Note that $\lim _{q \rightarrow 1} E_{n, q}^{(h)}=E_{n}$.

Let $T_{p}=\cup_{N \geq 1} C_{p^{N}}=\lim _{N \rightarrow \infty} C_{p^{N}}$, where $C_{p^{N}}=\left\{w \mid w^{p^{N}}=1\right\}$ is the cyclic group of order $p^{N}$. For $w \in T_{p}$, we denote by $\varphi_{w}: \mathbb{Z}_{p} \rightarrow \mathbb{C}_{p}$ the locally constant function $x \mapsto w^{x}$.

For $\alpha \in \mathbb{N}$ and $w \in T_{p}$, the twisted $q$-Euler numbers with weight $\alpha$ are also defined by

$$
\tilde{E}_{0, q, w}^{(\alpha)}=\frac{[2]_{q}}{w q+1}, \text { and } w q\left(q^{\alpha} \tilde{E}_{q, w}^{(\alpha)}+1\right)^{n}+\tilde{E}_{n, q, w}^{(\alpha)}= \begin{cases}{[2]_{q},} & \text { if } n=0 \\ 0, & \text { if } n>0\end{cases}
$$

with the usual convention about replacing $\left(\tilde{E}_{q, w}^{(\alpha)}\right)^{n}$ by $\tilde{E}_{n, q, w}^{(\alpha)}($ see $[2,5])$.

The main purpose of this article is to present a systemic study of some families of higherorder-twisted $q$-Euler numbers and polynomials with weight $\alpha$. In Section 2 , we investigate higher-order-twisted $q$-Euler numbers and polynomials with weight $\alpha$ and establish interesting properties. In Sections 3, 4, and 5, we observe some properties for special cases.

\section{Higher-order-twisted $\boldsymbol{q}$-Euler numbers and polynomials with weight $\alpha$}

For $h \in \mathbb{Z}, \alpha, k \in \mathbb{N}, w \in T_{p}$ and $n \in \mathbb{Z}_{+}$, let us consider the expansion of higherorder-twisted $q$-Euler polynomials with weight $\alpha$ as follows:

$$
\begin{aligned}
& \tilde{E}_{n, q, w}^{(\alpha)}(h, k \mid x) \\
& =\underbrace{\int_{\mathbb{Z}_{p}} \cdots \int_{\mathbb{Z}_{p}} w}_{k \text {-times }} \sum_{i=1}^{k} x_{i}\left[\sum_{i=1}^{k} x_{i}+x\right]_{q^{\alpha}}^{n} q^{x_{1}(h-1)+\cdots+x_{k}(h-k)} d \mu_{-q}\left(x_{1}\right) \cdots d \mu_{-q}\left(x_{k}\right) .
\end{aligned}
$$

From (1) and (3), we note that

$$
\tilde{E}_{n, q, w}^{(\alpha)}(h, k \mid x)=\frac{[2]_{q}^{k}}{\left(1-q^{\alpha}\right)^{n}} \sum_{l=0}^{n}\left(\begin{array}{l}
n \\
l
\end{array}\right)(-1)^{l} \frac{q^{\alpha l x}}{\left(1+w q^{\alpha l+h}\right) \cdots\left(1+w q^{\alpha l+h-k+1}\right)} .
$$


In the special case, $x=0 \tilde{E}_{n, q, w}^{(\alpha)}(h, k \mid 0)=\tilde{E}_{n, q, w}^{(\alpha)}(h, k)$ are called the higher-ordertwisted $q$-Euler numbers with weight $\alpha$.

By (3), we get

$$
\tilde{E}_{n, q, w}^{(\alpha)}(h, k)=\left(q^{\alpha}-1\right) \tilde{E}_{n+1, q, w}^{(\alpha)}(h-\alpha, k)+\tilde{E}_{n, q, w}^{(\alpha)}(h-\alpha, k) .
$$

From (5) and mathematical induction, we get the following theorem.

Theorem 1. For $\alpha, k \in \mathbb{N}$ and $n \in \mathbb{Z}_{+}$, we have

$$
\begin{aligned}
& \sum_{i=0}^{n-1}(-1)^{i-1}\left(q^{\alpha}-1\right)^{n-1-i} \tilde{E}_{n-i, q, w}^{(\alpha)}(h, k) \\
& =\left(q^{\alpha}-1\right)^{n-1} \tilde{E}_{n, q, w}^{(\alpha)}(h-\alpha, k)+(-1)^{n} E_{1, q, w}^{(\alpha)}(h-\alpha, k) .
\end{aligned}
$$

For complex number $q \in \mathbb{C}_{p}, m \in \mathbb{Z}_{+}$, we get the following;

$$
\begin{aligned}
q^{\alpha\left(x_{1}+\cdots+x_{k+1}\right) m} & =\left(1-\left(1-q^{\alpha\left(x_{1}+\cdots+x_{k+1}\right)}\right)\right)^{m} \\
& =\sum_{l=0}^{m}\left(\begin{array}{l}
m \\
l
\end{array}\right)(-1)^{l}\left(1-q^{\alpha\left(x_{1}+\cdots+x_{k+1}\right)}\right)^{l} \\
& =\sum_{l=0}^{m}\left(\begin{array}{l}
m \\
l
\end{array}\right)(-1)^{l}\left(1-q^{\alpha}\right)^{l} \frac{\left(1-q^{\alpha\left(x_{1}+\cdots+x_{k+1}\right)}\right)^{l}}{\left(1-q^{\alpha}\right)^{l}} \\
& =\sum_{l=0}^{m}\left(\begin{array}{l}
m \\
l
\end{array}\right)(-1)^{l}\left(1-q^{\alpha}\right)^{l}\left[x_{1}+x_{2}+\cdots+x_{k+1}\right]_{q^{\alpha}}^{l} .
\end{aligned}
$$

From (3), (4), and above property, we have

$$
\begin{aligned}
& \tilde{E}_{0, q, w}^{(\alpha)}(m \alpha, k+1) \\
& =\int_{\mathbb{Z}_{p}} \cdots \int_{\mathbb{Z}_{p}} w^{\sum_{j=1}^{k+1} x_{j}} q^{\sum_{j=1}^{k+1}(m \alpha-j) x_{j}} d \mu_{-q}\left(x_{1}\right) \cdots d \mu_{-q}\left(x_{k+1}\right) \\
& =\sum_{l=0}^{m}\left(\begin{array}{c}
m \\
l
\end{array}\right)\left(q^{\alpha}-1\right)^{l} \int_{\mathbb{Z}_{p}} \cdots \int_{\mathbb{Z}_{p}} w^{\sum_{j=1}^{k+1} x_{j}}\left[\sum_{j=1}^{k+1} x_{j}\right]_{q^{\alpha}}^{l} q^{-\sum_{j=1}^{k+1} j x_{j}} d \mu_{-q}\left(x_{1}\right) \cdots d \mu_{-q}\left(x_{k+1}\right) \\
& =\sum_{l=0}^{m}\left(\begin{array}{c}
m \\
l
\end{array}\right)\left(q^{\alpha}-1\right)^{l} \tilde{E}_{l, q, w}^{(\alpha)}(0, k+1) \\
& =\frac{[2]_{q}^{k+1}}{\left(1+w q^{\alpha m}\right)\left(1+w q^{\alpha m-1}\right) \ldots\left(1+w q^{\alpha m-k}\right)} .
\end{aligned}
$$

From (3), we can derive the following equation.

$$
\begin{aligned}
& \sum_{j=0}^{i}\left(\begin{array}{l}
i \\
j
\end{array}\right)\left(q^{\alpha}-1\right)^{j} \int_{\mathbb{Z}_{p}} \cdots \int_{\mathbb{Z}_{p}} w^{\sum_{s=1}^{k} x_{s}}\left[\sum_{s=1}^{k} x_{s}\right]_{q^{\alpha}}^{n-i+j} q^{\sum_{s=1}^{k}(h-\alpha-s) x_{s}} d \mu_{-q}\left(x_{1}\right) \cdots d \mu_{-q}\left(x_{k}\right) \\
& =\int_{\mathbb{Z}_{p}} \cdots \int_{\mathbb{Z}_{p}} w^{\sum_{s=1}^{k} x_{s}}\left[\sum_{s=1}^{k} x_{s}\right]_{q^{\alpha}}^{n-i} q^{\sum_{s=1}^{k}(h-s) x_{s}} q^{\alpha\left(\sum_{s=1}^{k} x_{s}\right)(i-1)} d \mu_{-q}\left(x_{1}\right) \cdots d \mu_{-q}\left(x_{k}\right) \\
& =\sum_{j=0}^{i-1}\left(q^{\alpha}-1\right)^{j}\left(\begin{array}{c}
i-1 \\
j
\end{array}\right) \tilde{E}_{n-i+j, q, w}^{(\alpha)}(h, k) .
\end{aligned}
$$


By (3), (4), (5), and (6), we see that

$$
\sum_{j=0}^{i}\left(q^{\alpha}-1\right)^{j}\left(\begin{array}{l}
i \\
j
\end{array}\right) \tilde{E}_{n-1+j, q, w}^{(\alpha)}(h-\alpha, k)=\sum_{j=0}^{i-1}\left(q^{\alpha}-1\right)^{j}\left(\begin{array}{c}
i-1 \\
j
\end{array}\right) \tilde{E}_{n-i+j, q, w}^{(\alpha)}(h, k) .
$$

Therefore, we obtain the following theorem.

Theorem 2. For $\alpha, k \in \mathbb{N}$ and $n, i \in \mathbb{Z}_{+}$, we have

$$
\sum_{j=0}^{i}\left(\begin{array}{l}
i \\
j
\end{array}\right)\left(q^{\alpha}-1\right)^{j} \tilde{E}_{n-i+j, q, w}^{(\alpha)}(h-\alpha, k)=\sum_{j=0}^{i-1}\left(q^{\alpha}-1\right)^{j}\left(\begin{array}{c}
i-1 \\
j
\end{array}\right) \tilde{E}_{n-i+j, q, w}^{(\alpha)}(h, k) .
$$

By simple calculation, we easily see that

$$
\sum_{j=0}^{m}\left(\begin{array}{c}
m \\
j
\end{array}\right)\left(q^{\alpha}-1\right)^{j} \tilde{E}_{j, q, w}^{(\alpha)}(0, k)=\frac{[2]_{q}^{k}}{\left(1+w q^{\alpha m}\right)\left(1+w q^{\alpha m-1}\right) \cdots\left(1+w q^{\alpha m-k+1}\right)}
$$

\section{Polynomials $\tilde{E}_{n, q, w}^{(\alpha)}(0, k \mid x)$}

We now consider the polynomials $\tilde{E}_{n, q, w}^{(\alpha)}(0, k \mid x)$ (in $\left.q^{x}\right)$ by

$$
\begin{aligned}
& \tilde{E}_{n, q, w}^{(\alpha)}(0, k \mid x) \\
& =\underbrace{\int_{\mathbb{Z}_{p}} \cdots \int_{\mathbb{Z}_{p}}}_{k-\text { times }} w^{x_{1}+\cdots+x_{k}}\left[x+\sum_{i=1}^{k} x_{i}\right]_{q^{\alpha}}^{n} q^{-\sum_{j=1}^{k} j x_{j}} d \mu_{-q}\left(x_{1}\right) \cdots d \mu_{-q}\left(x_{k}\right) .
\end{aligned}
$$

By (8) and (4), we get

$$
\left(q^{\alpha}-1\right)^{n} \tilde{E}_{n, q, w}^{(\alpha)}(0, k \mid x)=[2]_{q}^{k} \sum_{l=0}^{n}\left(\begin{array}{l}
n \\
l
\end{array}\right) q^{\alpha l x}(-1)^{n-1} \frac{1}{\left(1+w q^{\alpha l}\right) \cdots\left(1+w q^{\alpha l-k+1}\right)} .
$$

From (8) and (9), we can derive the following equation.

$$
\begin{aligned}
& \int_{\mathbb{Z}_{p}} \cdots \int_{\mathbb{Z}_{p}} w^{x_{1}+\cdots+x_{k}} q^{\sum_{j=1}^{k}(\alpha n-j) x_{j}+\alpha n x} d \mu_{-q}\left(x_{1}\right) \cdots d \mu_{-q}\left(x_{k}\right) \\
& =\sum_{j=0}^{n}\left(\begin{array}{c}
n \\
j
\end{array}\right)[\alpha]_{q}^{j}(q-1)^{j} \tilde{E}_{j, q, w}^{(\alpha)}(0, k \mid x),
\end{aligned}
$$

and

$$
\begin{aligned}
& \int_{\mathbb{Z}_{p}} \cdots \int_{\mathbb{Z}_{p}} w^{x_{1}+\cdots+x_{k}} q^{\sum_{j=1}^{k}(\alpha n-j) x_{j}+\alpha n x} d \mu_{-q}\left(x_{1}\right) \cdots d \mu_{-q}\left(x_{k}\right) \\
& =\frac{[2]_{q}^{k} q^{\alpha n x}}{\left(1+w q^{\alpha n}\right) \cdots\left(1+w q^{\alpha n-k+1}\right)} .
\end{aligned}
$$

Therefore, by (9) and (10), we obtain the following theorem.

Theorem 3. For $\alpha \in \mathbb{N}$ and $n, k \in \mathbb{Z}_{+}$, we have

$$
\tilde{E}_{n, q, w}^{(\alpha)}(0, k \mid x)=\frac{[2]_{q}^{k}}{[\alpha]_{q}^{n}(1-q)^{n}} \sum_{l=0}^{n}\left(\begin{array}{c}
n \\
l
\end{array}\right)(-1)^{l} q^{\alpha l x} \frac{1}{\left(-w q^{\alpha l-k+1}: q\right)_{k}}
$$


and

$$
\sum_{l=0}^{n}\left(\begin{array}{l}
n \\
l
\end{array}\right)[\alpha]_{q}^{l}(q-1)^{l} \tilde{E}_{l, q, w}^{(\alpha)}(0, k \mid x)=\frac{q^{\alpha n x}[2]_{q}^{k}}{\left(-w q^{\alpha n-k+1}: q\right)_{k}},
$$

where $(a: q)_{0}=1$ and $(a: q)_{k}=(1-a)(1-a q) \ldots\left(1-a q^{k-1}\right)$.

Let $d \in \mathbb{N}$ with $d \equiv 1(\bmod 2)$. Then we have

$$
\begin{aligned}
& \int_{\mathbb{Z}_{p}} \cdots \int_{\mathbb{Z}_{p}} w^{x_{1}+\cdots+x_{k}}\left[x+\sum_{j=1}^{k} x_{j}\right]_{q^{\alpha}}^{n} q^{-\sum_{j=1}^{k} j x_{j}} d \mu_{-q}\left(x_{1}\right) \cdots d \mu_{-q}\left(x_{k}\right) \\
& =\frac{[d]_{q^{\alpha}}^{n}}{[d]_{-q}^{k}} \sum_{a_{1}, \cdots, a_{k}=0}^{d-1} w^{a_{1}+\cdots+a_{k}} q^{-\sum_{j=2}^{k}(j-1) a_{j}}(-1)^{\sum_{j=1}^{k} a_{j} \times} \\
& \quad \int_{\mathbb{Z}_{p}} \cdots \int_{\mathbb{Z}_{p}} w^{d\left(x_{1}+\cdots+x_{k}\right)}\left[\frac{x+\sum_{j=1}^{k} a_{j}}{d}+\sum_{j=1}^{k} x_{j}\right]_{q^{\alpha d}}^{n} q^{-d \sum_{j=1}^{k} j x_{j}} d \mu_{-q^{d}}\left(x_{1}\right) \cdots d \mu_{-q^{d}}\left(x_{k}\right)
\end{aligned}
$$

Thus, by (11), we obtain the following theorem.

Theorem 4. For $d \in \mathbb{N}$ with $d \equiv 1(\bmod 2)$, we have

$$
\begin{aligned}
& \tilde{E}_{n, q, w}^{(\alpha)}(0, k \mid x) \\
& =\frac{[d]_{q^{\alpha}}^{n}}{[d]_{-q}^{k}} \sum_{a_{1}, \cdots, a_{k}=0}^{d-1}(-w)^{a_{1}+\cdots+a_{k}} q^{-\sum_{j=2}^{k}(j-1) a_{j}} \tilde{E}_{n, q^{d}, w^{d}}^{(\alpha)}\left(0, k \mid \frac{x+a_{1}+\cdots+a_{k}}{d}\right) .
\end{aligned}
$$

Moreover,

$$
\begin{aligned}
& \tilde{E}_{n, q, w}^{(\alpha)}(0, k \mid d x) \\
& =\frac{[d]_{q^{\alpha}}^{n}}{[d]_{-q}^{k}} \sum_{a_{1}, \cdots, a_{k}=0}^{d-1}(-w)^{a_{1}+\cdots+a_{k}} q^{-\sum_{j=2}^{k}(j-1) a_{j}} \tilde{E}_{n, q^{d}, w^{d}}^{(\alpha)}\left(0, k \mid x+\frac{a_{1}+\cdots+a_{k}}{d}\right) .
\end{aligned}
$$

By (8), we get

$$
\begin{aligned}
\tilde{E}_{n, q, w}^{(\alpha)}(0, k \mid x & =\sum_{l=0}^{n}\left(\begin{array}{c}
n \\
l
\end{array}\right)[x]_{q^{\alpha}}^{n-l} q^{\alpha l x} \tilde{E}_{l, q, w}^{(\alpha)}(0, k) \\
& =\left([x]_{q^{\alpha}}+q^{\alpha x} \tilde{E}_{q, w}^{(\alpha)}(0, k)\right)^{n},
\end{aligned}
$$

where $\tilde{E}_{n, q, w}^{(\alpha)}(0, k \mid 0)=\tilde{E}_{n, q, w}^{(\alpha)}(0, k)$.

Thus, we note that

$$
\begin{aligned}
\tilde{E}_{n, q, w}^{(\alpha)}(0, k \mid x+y & =\sum_{l=0}^{n}\left(\begin{array}{l}
n \\
l
\end{array}\right)[y]_{q^{\alpha}}^{n-l} q^{\alpha l y} \tilde{E}_{l, q, w}^{(\alpha)}(0, k \mid x) \\
& =\left([y]_{q^{\alpha}}+q^{\alpha \gamma} \tilde{E}_{q, w}^{(\alpha)}(0, k \mid x)\right)^{n} .
\end{aligned}
$$

4. Polynomials $\tilde{E}_{n, q, w}^{(\alpha)}(h, 1 \mid x)$

Let us define polynomials $\tilde{E}_{n, q, w}^{(\alpha)}(h, 1 \mid x)$ as follows:

$$
\tilde{E}_{n, q, w}^{(\alpha)}(h, 1 \mid x)=\int_{\mathbb{Z}_{p}} w^{x_{1}}\left[x+x_{1}\right]_{q^{\alpha}}^{n} q^{x_{1}(h-1)} d \mu_{-q}\left(x_{1}\right) .
$$


From (12), we have

$$
\tilde{E}_{n, q, w}^{(\alpha)}(h, 1 \mid x)=\frac{[2]_{q}}{\left(1-q^{\alpha}\right)^{n}} \sum_{l=0}^{n}\left(\begin{array}{l}
n \\
l
\end{array}\right)(-1)^{l} q^{\alpha l x} \frac{1}{\left(1+w q^{\alpha l+h}\right)} .
$$

By the calculation of the fermionic $p$-adic $q$-integral on $\mathbb{Z}_{p}$, we see that

$$
\begin{aligned}
& q^{\alpha x} \int_{\mathbb{Z}_{p}} w^{x_{1}}\left[x+x_{1}\right]_{q^{\alpha}}^{n} q^{x_{1}(h-1)} d \mu_{-q}\left(x_{1}\right) \\
& =\left(q^{\alpha}-1\right) \int_{\mathbb{Z}_{p}} w^{x_{1}}\left[x+x_{1}\right]_{q^{\alpha}}^{n+1} q^{x_{1}(h-\alpha-1)} d \mu_{-q}\left(x_{1}\right)+\int_{\mathbb{Z}_{p}} w^{x_{1}}\left[x+x_{1}\right]_{q^{\alpha}}^{n} q^{x_{1}(h-\alpha-1)} d \mu_{-q}\left(x_{1}\right) .
\end{aligned}
$$

Thus, by (13), we obtain the following theorem.

Theorem 5. For $\alpha \in \mathbb{N}$ and $h \in \mathbb{Z}$, we have

$$
q^{\alpha x} \tilde{E}_{n, q, w}^{(\alpha)}(h, 1 \mid x)=\left(q^{\alpha}-1\right) \tilde{E}_{n+1, q, w}^{(\alpha)}(h-\alpha, 1 \mid x)+\tilde{E}_{n, q, w}^{(\alpha)}(h-\alpha, 1 \mid x) .
$$

It is easy to show that

$$
\begin{aligned}
\tilde{E}_{n, q, w}^{(\alpha)}(h, 1 \mid x) & =\int_{\mathbb{Z}_{p}} w^{x_{1}}\left[x+x_{1}\right]_{q^{\alpha}}^{n} q^{x_{1}(h-1)} d \mu_{-q}\left(x_{1}\right) \\
& =\sum_{l=0}^{n}\left(\begin{array}{c}
n \\
l
\end{array}\right)[x]_{q^{\alpha}}^{n-1} q^{\alpha l x} \int_{\mathbb{Z}_{p}} w^{x_{1}}\left[x_{1}\right]_{q^{\alpha}}^{l} q^{x_{1}(h-1)} d \mu_{-q}\left(x_{1}\right) \\
& =\sum_{l=0}^{n}\left(\begin{array}{c}
n \\
l
\end{array}\right)[x]_{q^{\alpha}}^{n-1} q^{\alpha l x} \tilde{E}_{l, q, w}^{(\alpha)}(h, 1) \\
& =\left(q^{\alpha x} \tilde{E}_{q, w}^{(\alpha)}(h, 1)+[x]_{q^{\alpha}}\right)^{n}, \quad \text { for } n \geq 1,
\end{aligned}
$$

with the usual convention about replacing $\left(\tilde{E}_{q, w}^{(\alpha)}(h, 1)\right)^{n}$ by $\tilde{E}_{n, q, w}^{(\alpha)}(h, 1)$.

From $q I_{-q}\left(f_{1}\right)+I_{-q}(f)=[2]_{q} f(0)$, we have

$$
\begin{aligned}
& w q^{h} \int_{\mathbb{Z}_{p}} w^{x_{1}}\left[x+x_{1}+1\right]_{q^{\alpha}}^{n} q^{x_{1}(h-1)} d \mu_{-q}\left(x_{1}\right)+\int_{\mathbb{Z}_{p}} w^{x_{1}}\left[x+x_{1}\right]_{q^{\alpha}}^{n} q^{x_{1}(h-1)} d \mu_{-q}\left(x_{1}\right) \\
& =[2]_{q}[x]_{q^{\alpha}}^{n} .
\end{aligned}
$$

By (13) and (15), we get

$$
w q^{h} \tilde{E}_{n, q, w}^{(\alpha)}(h, 1 \mid x+1)+\tilde{E}_{n, q, w}^{(\alpha)}(h, 1 \mid x)=[2]_{q}[x]_{q^{\alpha}}^{n} .
$$

For $x=0$ in (16), we have

$$
w q^{h} \tilde{E}_{n, q, w}^{(\alpha)}(h, 1 \mid 1)+\tilde{E}_{n, q, w}^{(\alpha)}(h, 1)= \begin{cases}{[2]_{q},} & \text { if } n=0 \\ 0, & \text { if } n>0 .\end{cases}
$$

Therefore, by (14) and (17), we obtain the following theorem.

Theorem 6. For $h \in \mathbb{Z}$ and $n \in \mathbb{Z}_{+}$, we have

$$
w q^{h}\left(q^{\alpha} \tilde{E}_{q, w}^{(\alpha)}(h, 1)+1\right)^{n} \tilde{E}_{n, q, w}^{(\alpha)}(h, 1)= \begin{cases}{[2]_{q^{\prime}}} & \text { if } n=0, \\ 0, & \text { if } n>0,\end{cases}
$$

with the usual convention about replacing $\left(\tilde{E}_{q, w}^{(\alpha)}(h, 1)\right)^{n}$ by $\tilde{E}_{n, q, w}^{(\alpha)}(h, 1)$. 
From the fermionic $p$-adic $q$-integral on $\mathbb{Z}_{p}$, we easily get

$$
\tilde{E}_{0, q, w}^{(\alpha)}(h, 1)=\int_{\mathbb{Z}_{p}} w^{x_{1}} q^{x_{1}(h-1)} d \mu_{-q}\left(x_{1}\right)=\frac{[2]_{q}}{[2]_{w q^{h}}} .
$$

By (12), we see that

$$
\begin{aligned}
& \tilde{E}_{n, q^{-1}, w^{-1}}^{(\alpha)}(h, 1 \mid 1-x)=\int_{\mathbb{Z}_{p}} w^{-1}\left[1-x+x_{1}\right]_{q^{-\alpha}}^{n} q^{-x_{1}(h-1)} d \mu_{-q^{-1}}\left(x_{1}\right) \\
& =(-1)^{n} w q^{\alpha n+h-1} \frac{[2]_{q}}{\left(1-q^{\alpha}\right)^{n}} \sum_{l=0}^{n}\left(\begin{array}{c}
n \\
l
\end{array}\right)(-1)^{l} q^{\alpha l x} \frac{1}{1+w q^{\alpha l+h}} \\
& =(-1)^{n} w q^{\alpha n+h-1} \tilde{E}_{n, q, w}^{(\alpha)}(h, 1 \mid x)
\end{aligned}
$$

Therefore, by (18), we obtain the following theorem.

Theorem 7. For $\alpha \in \mathbb{N}, h \in \mathbb{Z}$ and $n \in \mathbb{Z}_{+}$, we have

$$
\tilde{E}_{n, q^{-1}, w^{-1}}^{(\alpha)}(h, 1 \mid 1-x)=(-1)^{n} w q^{\alpha n+h-1} \tilde{E}_{n, q, w}^{(\alpha)}(h, 1 \mid x) .
$$

In particular, for $x=1$, we get

$$
\begin{aligned}
& \tilde{E}_{n, q, w}^{(\alpha)}(h, 1) \\
& =(-1)^{n} w q^{\alpha n+h-1} \tilde{E}_{n, q, w}^{(\alpha)}(h, 1 \mid 1) \\
& =(-1)^{n+1} q^{\alpha n-1} \tilde{E}_{n, q, w}^{(\alpha)}(h, 1) \text { if } n \geq 1 .
\end{aligned}
$$

Let $d \in \mathbb{N}$ with $d \equiv 1(\bmod 2)$. Then we have

$$
\begin{aligned}
& \int_{\mathbb{Z}_{p}} w^{x_{1}} q^{x_{1}(h-1)}\left[x+x_{1}\right]_{q^{\alpha}}^{n} d \mu_{-q}\left(x_{1}\right) \\
& =\frac{[d]_{q^{\alpha}}^{n}}{[d]_{-q}} \sum_{a=0}^{d-1} w^{a} q^{h a}(-1)^{a} \int_{\mathbb{Z}_{p}} w^{d x_{1}}\left[\frac{x+a}{d}+x_{1}\right]_{q^{\alpha d}}^{n} q^{x_{1}(h-1) d} d \mu_{-q^{d}}\left(x_{1}\right) .
\end{aligned}
$$

Therefore, by (19), we obtain the following theorem.

Theorem 8 (Multiplication formula). For $d \in \mathbb{N}$ with $d \equiv 1(\bmod 2)$, we have

$$
\tilde{E}_{n, q, w}^{(\alpha)}(h, 1 \mid x)=\frac{[d]_{q^{\alpha}}^{n}}{[d]_{-q}} \sum_{a=0}^{d-1} w^{a} q^{h a}(-1)^{q} \tilde{E}_{n, q^{d}, w^{d}}^{(\alpha)}\left(h, 1 \mid \frac{x+a}{d}\right) .
$$

5. Polynomials $\tilde{E}_{n, q, w}^{(\alpha)}(h, k \mid x)$ and $\boldsymbol{k}=\boldsymbol{h}$

In (3), we know that

$$
\begin{aligned}
& \tilde{E}_{n, q, w}^{(\alpha)}(h, k \mid x) \\
& =\int_{\mathbb{Z}_{p}} \cdots \int_{\mathbb{Z}_{p}} w^{x_{1}+\cdots+x_{k}}\left[x_{1}+\cdots+x_{k}+x\right]_{q^{\alpha}}^{n} q^{(h-1) x_{1}+\cdots+(h-k) x_{k}} d \mu_{-q}\left(x_{1}\right) \cdots d \mu_{-q}\left(x_{k}\right) .
\end{aligned}
$$

Thus, we get

$$
\left(q^{\alpha}-1\right)^{n} \tilde{E}_{n, q, w}^{(\alpha)}(h, k \mid x)=[2]_{q}^{k} \sum_{l=0}^{n}\left(\begin{array}{l}
n \\
l
\end{array}\right)(-1)^{n-l} \frac{q^{\alpha l x}}{\left(1+w q^{\alpha l+h}\right) \cdots\left(1+w q^{\alpha l+h-k+1}\right)},
$$


and

$$
\begin{aligned}
& w q^{h} \int_{\mathbb{Z}_{p}} \cdots \int_{\mathbb{Z}_{p}} w^{x_{1}+\cdots+x_{k}}\left[x+1+\sum_{i=1}^{k} x_{i}\right]_{q^{\alpha}}^{n} q^{\sum_{i=1}^{k}(h-i) x_{i}} d \mu_{-q}\left(x_{1}\right) \cdots d \mu_{-q}\left(x_{k}\right) \\
& =-\int_{\mathbb{Z}_{p}} \cdots \int_{\mathbb{Z}_{p}} w^{x_{1}+\cdots+x_{k}}\left[x+\sum_{i=1}^{k} x_{i}\right]_{q^{\alpha}}^{n} q^{\sum_{i=1}^{k}(h-i) x_{i}} d \mu_{-q}\left(x_{1}\right) \cdots d \mu_{-q}\left(x_{k}\right) \\
& +[2]_{q} \int_{\mathbb{Z}_{p}} \cdots \int_{\mathbb{Z}_{p}} w^{x_{2}+\cdots+x_{k}}\left[x+\sum_{i=2}^{k} x_{i}\right]_{q^{\alpha}}^{n} q^{\sum_{i=2}^{k}(h-i) x_{i}} d \mu_{-q}\left(x_{2}\right) \cdots d \mu_{-q}\left(x_{k}\right) .
\end{aligned}
$$

Therefore, by (3) and (20), we obtain the following theorem.

Theorem 9. For $h \in \mathbb{Z}, \alpha \in \mathbb{N}$ and $n \in \mathbb{Z}_{+}$, we have

$$
w q^{h} \tilde{E}_{n, q, w}^{(\alpha)}(h, k \mid x+1)+\tilde{E}_{n, q, w}^{(\alpha)}(h, k \mid x)=[2]_{q} \tilde{E}_{n, q, w}^{(\alpha)}(h-1, k-1 \mid x) .
$$

Note that

$$
\begin{aligned}
& q^{\alpha x} \int_{\mathbb{Z}_{p}} \cdots \int_{\mathbb{Z}_{p}} w^{x_{1}+\cdots+w_{k}}\left[x+\sum_{i=1}^{k} x_{i}\right]_{q^{\alpha}}^{n} q^{\sum_{i=1}^{k}(h-i) x_{i}} d \mu_{-q}\left(x_{1}\right) \cdots d \mu_{-q}\left(x_{k}\right) \\
& =\left(q^{\alpha}-1\right) \int_{\mathbb{Z}_{p}} \cdots \int_{\mathbb{Z}_{p}} w^{x_{1}+\cdots+x_{k}}\left[x+\sum_{i=1}^{k} x_{i}\right]_{q^{\alpha}}^{n+1} q^{\sum_{i=1}^{k}(h-\alpha-i) x_{i}} d \mu_{-q}\left(x_{1}\right) \cdots d \mu_{-q}\left(x_{k}\right) \\
& \quad+\int_{\mathbb{Z}_{p}} \cdots \int_{\mathbb{Z}_{p}} w^{x_{1}+\cdots+x_{k}}\left[x+\sum_{i=1}^{k} x_{i}\right]_{q^{\alpha}}^{n} q^{\sum_{i=1}^{k}(h-\alpha-i) x_{i}} d \mu_{-q}\left(x_{1}\right) \cdots d \mu_{-q}\left(x_{k}\right) \\
& =\left(q^{\alpha}-1\right) \tilde{E}_{n+1, q, w}^{(\alpha)}(h-\alpha, k \mid x)+\tilde{E}_{n, q, w}^{(\alpha)}(h-\alpha, k \mid x) .
\end{aligned}
$$

Therefore, by (21), we obtain the following theorem.

Theorem 10. For $n \in \mathbb{Z}_{+}$, we have

$$
q^{\alpha x} \tilde{E}_{n, q, w}^{(\alpha)}(h, k \mid x)=\left(q^{\alpha}-1\right) \tilde{E}_{n+1, q, w}^{(\alpha)}(h-\alpha, k \mid x)+\tilde{E}_{n, q, w}^{(\alpha)}(h-\alpha, k \mid x) .
$$

Let $d \in \mathbb{N}$ with $d \equiv 1(\bmod 2)$. Then we get

$$
\begin{aligned}
& \int_{\mathbb{Z}_{p}} \cdots \int_{\mathbb{Z}_{p}} w^{x_{1}+\cdots+x_{k}}\left[x+\sum_{j=1}^{k} x_{j}\right]_{q^{\alpha}}^{n} q^{\sum_{j=1}^{k}(h-j) x_{j}} d \mu_{-q}\left(x_{1}\right) \cdots d \mu_{-q}\left(x_{k}\right) \\
& =\frac{[d]_{q^{\alpha}}^{n}}{[d]_{-q}^{k}} \sum_{a_{1}, \cdots, a_{k}=0}^{d-1} w^{a_{1}+\cdots+a_{k}} q^{h \sum_{j=1}^{k} a j-\sum_{j=2}^{k}(j-1) a_{j}}(-1)^{\sum_{j=1}^{k} a_{j} \times} \\
& \int_{\mathbb{Z}_{p}} \cdots \int_{\mathbb{Z}_{p}} w^{d\left(x_{1}+\cdots+x_{k}\right)}\left[\frac{x+\sum_{j=1}^{k} a_{j}}{d}+\sum_{j=1}^{k} x_{j}\right]_{q^{\alpha d}}^{n} q^{d \sum_{j=1}^{k}(h-j) x_{j}} d \mu_{-q^{d}}\left(x_{1}\right) \cdots d \mu_{-q^{d}}\left(x_{k}\right) .
\end{aligned}
$$

Therefore, by (22), we obtain the following theorem.

Theorem 11. For $d \in \mathbb{N}$ with $d \equiv 1(\bmod 2)$, we have

$$
\begin{aligned}
& \tilde{E}_{n, q, w}^{(\alpha)}(h, k \mid d x) \\
& =\frac{[d]_{q^{\alpha}}^{n}}{[d]_{-q}^{k}} \sum_{a_{1}, \cdots, a_{k}=0}^{d-1} w^{a_{1}+\cdots+a_{k}} q^{h \sum_{j=1}^{k} a j-\sum_{j=2}^{k}(j-1) a_{j}}(-1)^{\sum_{j=1}^{k} a_{j}} \tilde{E}_{n, q^{d}, w^{d}}^{(\alpha)}\left(h, k \mid x+\frac{a_{1}+\cdots+a_{k}}{d}\right) .
\end{aligned}
$$


Let $\tilde{E}_{n, q, w}^{(\alpha)}(k, k \mid x)=\tilde{E}_{n, q, w}^{(\alpha)}(k \mid x)$. Then we get

$$
\left(q^{\alpha}-1\right)^{n} \tilde{E}_{n, q, w}^{(\alpha)}(k \mid x)=\sum_{l=0}^{n}\left(\begin{array}{l}
n \\
l
\end{array}\right)(-1)^{n-l} q^{\alpha l x} \frac{[2]_{q}^{k}}{\left(1+w q^{\alpha l+k}\right) \cdots\left(1+w q^{\alpha l+1}\right)},
$$

and

$$
\begin{aligned}
& \int_{\mathbb{Z}_{p}} \cdots \int_{\mathbb{Z}_{p}} w^{-\left(x_{1}+\cdots+x_{k}\right)}\left[k-x+\sum_{i=1}^{k} x_{i}\right]_{q^{-\alpha}}^{n} q^{-\sum_{i=1}^{k}(k-i) x_{i}} d \mu_{-q^{-1}}\left(x_{1}\right) \cdots d \mu_{-q^{-1}}\left(x_{k}\right) \\
& =\frac{q\left(\begin{array}{c}
k \\
2
\end{array}\right)}{\left(1-q^{-\alpha}\right)^{n}}[2]_{q}^{k} \sum_{l=0}^{n}\left(\begin{array}{c}
n \\
l
\end{array}\right)(-1)^{l} q^{\alpha l x} \frac{1}{\left(1+w q^{\alpha l+1}\right) \cdots\left(1+w q^{\alpha l+k}\right)} \\
& =(-1)^{n} q^{n \alpha} q^{\left(\begin{array}{l}
k \\
2
\end{array}\right)} \frac{[2]_{q}^{k}}{\left(1-q^{\alpha}\right)^{n}} \sum_{l=0}^{n} \frac{\left(\begin{array}{c}
n \\
l
\end{array}\right)(-1)^{l} q^{\alpha l x}}{\left(1+w q^{\alpha l+1}\right) \cdots\left(1+w q^{\alpha l+k}\right)} \\
& =(-1)^{n} q
\end{aligned}
$$

Therefore, by (23), we obtain the following theorem.

Theorem 12. For $n \in \mathbb{Z}_{+}$, we have

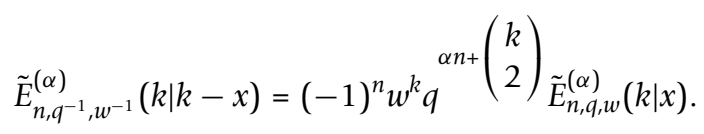

Let $x=k$ in Theorem 12. Then we see that

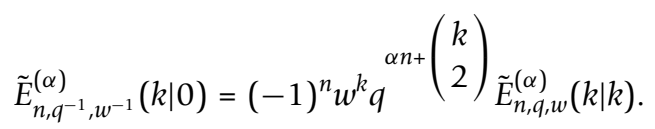

From (15), we note that

$$
w q^{k} \tilde{E}_{n, q, w}^{(\alpha)}(k \mid x+1)+\tilde{E}_{n, q, w}^{(\alpha)}(k \mid x)=[2]_{q} \tilde{E}_{n, q, w}^{(\alpha)}(k-1 \mid x) .
$$

It is easy to show that

$$
\left(q^{\alpha}-1\right)^{n} \tilde{E}_{n, q, w}^{(\alpha)}(k \mid 0)=\sum_{l=0}^{n}\left(\begin{array}{l}
n \\
l
\end{array}\right)(-1)^{l+n} \frac{[2]_{q}^{k}}{\left(1+w q^{\alpha l+1}\right) \cdots\left(1+w q^{\alpha l+k}\right)} .
$$

By simple calculation, we get

$$
\begin{aligned}
& \sum_{l=0}^{n}\left(\begin{array}{c}
n \\
l
\end{array}\right)\left(q^{\alpha}-1\right)^{l} \int_{\mathbb{Z}_{p}} \cdots \int_{\mathbb{Z}_{p}} w^{\sum_{i=1}^{k} x_{k}}\left[\sum_{i=1}^{k} x_{k}\right]_{q^{\alpha}}^{l} q^{\sum_{l=i}^{k}(k-i) x_{i}} d \mu_{-q}\left(x_{1}\right) \cdots d \mu_{-q}\left(x_{k}\right) \\
& =\frac{[2]_{q}^{k}}{\left(1+w q^{\alpha n+k}\right)\left(1+w q^{\alpha n+k-1}\right) \cdots\left(1+w q^{\alpha+1}\right)} .
\end{aligned}
$$

From (26), we note that

$$
\sum_{l=0}^{n}\left(\begin{array}{c}
n \\
l
\end{array}\right)\left(q^{\alpha}-1\right)^{l} \tilde{E}_{l, q, w}^{(\alpha)}(k \mid 0)=\frac{[2]_{q}^{k}}{\left(1+w q^{\alpha n+k}\right)\left(1+w q^{\alpha n+k-1}\right) \cdots\left(1+w q^{\alpha n+1}\right)},
$$


and

$$
\begin{aligned}
\tilde{E}_{n, q, w}^{(\alpha)}(k \mid x) & =\int_{\mathbb{Z}_{p}} \cdots \int_{\mathbb{Z}_{p}} w^{\sum_{i=1}^{k} x_{k}}\left[x+\sum_{i=1}^{k} x_{k}\right]_{q^{\alpha}}^{n} q^{\sum_{i=1}^{k}(k-i) x_{i}} d \mu_{-q}\left(x_{1}\right) \cdots d \mu_{-q}\left(x_{k}\right) \\
& =\sum_{l=0}^{n}\left(\begin{array}{c}
n \\
l
\end{array}\right)[x]_{q^{\alpha}}^{n-l} q^{\alpha l x} \tilde{E}_{l, q, w}^{(\alpha)}(k \mid 0) \\
& =\left(q^{x \alpha} \tilde{E}_{q, w}^{(\alpha)}(k \mid 0)+[x]_{q^{\alpha}}\right)^{n}, n \in \mathbb{Z}_{+,}
\end{aligned}
$$

with the usual convention about replacing $\left(\tilde{E}_{q, w}^{(\alpha)}(k \mid 0)\right)^{n}$ by $\tilde{E}_{n, q, w}^{(\alpha)}(k \mid 0)$.

Put $x=0$ in (25), we get

$$
w q^{k} \tilde{E}_{n, q, w}^{(\alpha)}(k \mid 1)+\tilde{E}_{n, q, w}^{(\alpha)}(k \mid 0)=[2]_{q} \tilde{E}_{n, q}^{(\alpha)}(k-1 \mid 0) .
$$

Thus, we have

$$
w q^{k}\left(q^{\alpha} \tilde{E}_{q, w}^{(\alpha)}(k \mid 0)+1\right)^{n}+\tilde{E}_{n, q, w}^{(\alpha)}(k \mid 0)=[2]_{q} \tilde{E}_{n, q, w}^{(\alpha)}(k-1 \mid 0),
$$

with the usual convention about replacing $\left(\tilde{E}_{q, w}^{(\alpha)}(k \mid 0)\right)^{n}$ by $\tilde{E}_{n, q, w}^{(\alpha)}(k \mid 0)$.

\section{Acknowledgements}

The authors express their gratitude to the referee for his/her valuable comments.

\section{Authors' contributions}

All authors contributed equally to the manuscript and read and approved the final manuscript.

\section{Competing interests}

The authors declare that they have no competing interests.

\section{Received: 19 September 2011 Accepted: 29 February 2012 Published: 29 February 2012}

\section{References}

1. Ryoo, CS: On the generalized Barnes type multiple $q$-Euler polynomials twisted by ramified roots of unity. Proc Jangjeon Math Soc. 13, 255-263 (2010)

2. Ryoo, CS: A note on the weighted q-Euler numbers and polynomials. Adv Stud Contemp Math. 21, 47-54 (2011)

3. Moon, E-J, Rim, S-H, Jin, J-H, Lee, S-J: On the symmetric properties of higher-order twisted $q$-Euler numbers and polynomials. Adv Diff Equ 2010, 8 (2010). (Art ID 765259)

4. Cangul, IN, Kurt, V, Ozden, H, Simsek, Y: On the higher-order w-q-Genocchi numbers. Adv Stud Contemp Math. 19, 39-57 (2009)

5. Jang, LC: A note on Nörlund-type twisted q-Euler polynomials and numbers of higher order associated with fermionic invariant q-integrals. J Inequal Appl 2010, 12 (2010). (Art ID 417452)

6. Kim, T: q-Volkenborn integration. Russ J Math Phys. 9, 288-299 (2002)

7. Kim, T: A note on q-Volkenborn integration. Proc Jangjeon Math Soc. 8, 13-17 (2005)

8. Kim, T, Choi, J, Kim, YH: On extended Carlitz's type q-Euler numbers and polynomials. Adv Stud Contemp Math. 20, 499-505 (2010)

9. Kim, T: The modified q-Euler numbers and polynomials. Adv Stud Contemp Math. 16, 161-170 (2008)

10. Kim, T: q-Euler numbers and polynomials associated with $p$-adic q-integrals. J Nonlinear Math Phys. 14, 15-27 (2007). doi:10.2991/jnmp.2007.14.1.3

11. Kim, T: Note on the Euler q-zeta functions. J Number Theory. 129, 1798-1804 (2009). doi:10.1016/j.jnt.2008.10.007

12. Kim, T: Some identities on the $q$-Euler polynomials of higher order and $q$-Stirling numbers by the fermionic $p$-adic integral on $\bigotimes_{p}$. Russ J Math Phys. 16, 484-491 (2009). doi:10.1134/S1061920809040037

13. Kim, T: Barnes type multiple q-zeta function and q-Euler polynomials. J Phys A: Math Theor 43, 11 (2010). (Art ID 255201)

14. Kim, T, Choi, J, Kim, YH, Ryoo, CS: On the fermionic $p$-adic integral representation of Bernstein polynomials associated with Euler numbers and polynomials. J Inequal Appl 2010, 12 (2010). (Art ID 864247)

15. Kim, T, Choi, J, Kim, YH, Ryoo, CS: A note on the weighted $p$-adic q-Euler measure on $\bigotimes_{p}$. Adv Stud Contemp Math. 21, 35-40 (2011)

16. Kurt, V: A further symmetric relation on the analogue of the Apostol-Bernoulli and the analogue of the ApostolGenocchi polynomials. Appl Math Sci. 3, 53-56 (2009) 